\title{
Endothelial and nonendothelial sources of PDGF-B regulate pericyte recruitment and influence vascular pattern formation in tumors
}

\author{
Alexandra Abramsson, Per Lindblom, and Christer Betsholtz \\ Department of Medical Biochemistry, Sahlgrenska Academy at Göteborg University, Göteborg, Sweden
}

\begin{abstract}
Tumor-infiltrating blood vessels deviate morphologically and biochemically from normal vessels, raising the prospect of selective pharmacological targeting. Current antiangiogenic approaches focus mainly on endothelial cells, but recent data imply that targeting pericytes may provide additional benefits. Further development of these concepts will require deeper insight into mechanisms of pericyte recruitment and function in tumors. Here, we applied genetic tools to decipher the function of PDGF-B and PDGF-R $\beta$ in pericyte recruitment in a mouse fibrosarcoma model. In tumors transplanted into PDGF-B retention motif-deficient ( $\left.p d g f-b^{\text {ret} / \mathrm{ret}}\right)$ mice, pericytes were fewer and were partially detached from the vessel wall, coinciding with increased tumor vessel diameter and hemorrhaging. Transgenic PDGF-B expression in tumor cells was able to increase the pericyte density in both WT and $p d g f-b^{\mathrm{ret} / \mathrm{ret}}$ mice but failed to correct the pericyte detachment in $p d g f-b^{\mathrm{ret} / \mathrm{ret}}$ mice. Coinjection of exogenous pericytes and tumor cells showed that pericytes require PDGF-R $\beta$ for recruitment to tumor vessels, whereas endothelial PDGF-B retention is indispensable for proper integration of pericytes in the vessel wall. Our data support the notion that pericytes serve an important function in tumor vessels and highlight PDGF-B and PDGF-R $\beta$ as promising molecular targets for therapeutic intervention.
\end{abstract}

J. Clin. Invest. 112:1142-1151 (2003). doi:10.1172/JCI200318549.

\begin{abstract}
Introduction
Most solid tumors induce a neovascular response in order to secure a sufficient supply of nutrients and oxygen (1). However, the vessels formed to support tumor enlargement show many signs of structural heterogeneity and dysfunction, including lack of a hierarchical branching organization, irregular diameter, and increased leakiness (2-6). The cell and ECM composition of tumor vessels is also abnormal; some tumor vessels have an incomplete basement membrane, and typically the pericyte coverage is low and irregular (7-13). The causes of these abnormalities are not known, but at least part of the tumor vessel phenotype is believed to reflect lack of proper maturation $(14,15)$. Because of their critical role for tumor growth, tumor blood vessels are recognized as potential antitumor drug targets, and their abnormalities open up the possibility of targeting tumor vessels separately from the vessels of the surrounding normal tissue (16-18).
\end{abstract}

Received for publication April 3, 2003, and accepted in revised form September 16, 2003.

Address correspondence to: Christer Betsholtz, Department of Medical Biochemistry, University of Göteborg, P.O. Box 440, SE 40530 Göteborg, Sweden. Phone: 46-31-7733460;

Fax: 46-31-416108; E-mail: Christer.Betsholtz@medkem.gu.se.

Conflict of interest: The authors have declared that no conflict of interest exists.

Nonstandard abbreviations used: receptor tyrosine kinase (RTK); murine embryonic fibroblast (MEF); paraformaldehyde (PFA); $\alpha$-smooth muscle actin (SMA).
Pericytes are considered to be required for normal microvascular stability and function (19). Pericyte deficiency, as seen in mice lacking PDGF-B and its cognate receptor PDGF-R $\beta$, promotes a range of microvascular changes, such as endothelial hyperplasia, vessel dilation, tortuosity, leakage, and rupture, leading to widespread and lethal microhemorrhage and edema at late gestation (20-22). Models that allow postnatal analysis of pericyte recruitment defects have further demonstrated a strict requirement for pericytes in shaping a functional retinal vasculature (23-25). Thus, pericytes are critically involved in normal developmental microvessel formation in many organs, and the pericyte-deficient state caused by genetic ablation of PDGF-B or PDGF-R $\beta$ leads to the formation of microvessels with many of the typical hallmarks of tumor vessels.

In analogy with developmental angiogenesis, the extent of pericyte recruitment in tumor angiogenesis likely has important functional implications. The heterogeneity in pericyte density and investment commonly observed between and within tumors may correlate with the structural irregularities and functional heterogeneity observed. Intriguingly, normal vessels can tolerate substantial reduction in the density of pericytes. Reduction of up to $90 \%$ of the pericyte coverage in mice, while causing structural and functional abnormalities in the microvasculature, is compatible with embryonic and postnatal survival (24). Loss of more than $95 \%$ of the pericytes, however, is lethal $(20,24)$. This suggests that a rather low threshold density of pericytes is 
required for basal microvascular function. Thus, even small numbers of pericytes in tumor vessels may be critical for vessel integrity and function. This view is supported by studies showing that tumor vessels lacking pericytes are more dependent on VEGF for their survival than are vessels invested by pericytes (12), suggesting that the efficiency of an antitumor therapy directed against VEGF signaling may correlate inversely with the propensity of the tumor to recruit pericytes. A recent study demonstrated that pericyte density correlated with the effect of antiangiogenic therapy also when this was directed against targets other than VEGF (15). Tyrosine kinase inhibitors affecting multiple receptor tyrosine kinases (RTKs) may exert their antitumor activity in part by reducing pericyte density in the tumor vessels, thereby sensitizing them to inhibition of endothelial RTKs (10). Combinations of RTK inhibitors targeting kinases in both endothelial cells and pericytes were recently shown to inhibit the growth of mouse insulinomas better than any of the RTK blockers individually (26). Together, these observations suggest that pericytes may constitute a relevant target cell type for antitumor angiogenesis therapy.

If pericytes are considered as a target for tumor therapy, it becomes important to map the molecular mechanisms involved in pericyte recruitment to the tumor vessel wall. Previous studies have demonstrated that pericyte recruitment in developmental angiogenesis depends on endothelium-derived PDGF-B and pericytic expression of PDGF-R $\beta(20,21)$. It is unclear, however, to what extent the same mechanisms are involved in pathological angiogenesis. Recent experiments suggest that PDGF-B expression stimulates pericyte recruitment in gliomas (27). Using kinase inhibitors, a functional role for PDGF-R $\beta$ has also been implicated in pericyte recruitment in mouse insulinomas (26). However, these agents inhibit a broad range of kinases, and it cannot be ruled out that their effects on pericyte recruitment result from the inhibition of kinases other than PDGF-R $\beta$. PDGF-B and PDGF-R $\beta$ also have other roles besides pericyte recruitment that may affect tumor growth. PDGF-B expression is commonly observed in several types of tumors and has been implicated in autocrine growth stimulation, the formation of tumor stroma, and the control of interstitial tumor pressure (28-31).

In the present study, we address the role of paracrine PDGF-B/PDGF-R $\beta$ signaling in tumor vessel pericyte recruitment using a set of specific genetic tools. We show that PDGF-R $\beta$ expression by pericytes is necessary for their recruitment to tumor vessels and that extracellular retention of PDGF-B produced by the tumor endothelium is required for the recruitment of adequate numbers of pericytes, as well as for proper integration of pericytes in the vascular wall. We also show that PDGF-B expression from the tumor cells enhances tumor pericyte recruitment. Finally, our studies show that impaired pericyte recruitment correlates with increased tumor vessel diameter.

\section{Methods}

Animals, cell culture, and tumor transplantation. Tumor cells and murine embryonic fibroblasts (MEFs) were cultured and transplanted as described (13). Tumors were studied in WT C57BL6 $(n=7)$ mice or transgenic mice lacking the PDGF-B retention motif ( $p d g f-b^{\text {ret } / \text { ret }}$ mice) $(n=5)$ backcrossed on the C57BL6 genetic background. The $p d g f-b^{\text {ret} / r e t ~}$ mice were identified by genotyping tail DNA by PCR, which was performed using four primers that specifically recognized the WT and mutated alleles, respectively. Forward $5^{\prime}$-CATGCTGCCTTGTAATCCGTTC$3^{\prime}$ and reverse $5^{\prime}$-GGCGGATTCTCACCGT- $3^{\prime}$ produced a 340-bp WT fragment, and forward $5^{\prime}$-GGTGACCATTCGGTAA- $3^{\prime}$ and reverse $5^{\prime}$-TCTAAGTCACAGCCAGGGAGTAGC- $3^{\prime}$ produced a 212 -bp fragment from the $p d g f-b^{\text {ret }}$ allele. The PCR reactions contained $2 \mu \mathrm{l} 10 \times \mathrm{PCR}$ buffer (200 mM Tris-HCl, pH 8.4, and $500 \mathrm{mM} \mathrm{KCl}$ ), $1 \mu$ DNA, $0.2 \mu \mathrm{M}$ primer, $0.2 \mathrm{mM}$ dNTP's, $1 \mathrm{U}$ Taq polymerase, and $1.5 \mathrm{mM} \mathrm{MgCl}_{2}$ in a $20-\mu \mathrm{l}$ volume. Reactions were completed on a model 9700 thermal cycler from PE Biosystems (Foster City, California, USA) using this program: seven cycles of $95^{\circ} \mathrm{C}$ for 5 minutes, $94^{\circ} \mathrm{C}$ for 30 seconds, $60^{\circ} \mathrm{C}$ (with the temperature decreasing $0.5^{\circ} \mathrm{C}$ per cycle) for 45 seconds, and $72^{\circ} \mathrm{C}$ for 90 seconds, followed by 27 cycles of $94^{\circ} \mathrm{C}$ for 45 seconds, $55^{\circ} \mathrm{C}$ for 45 seconds, and $72^{\circ} \mathrm{C}$ for 90 seconds.

Animals were housed under barrier conditions in the transgenic core facility of Göteborg University. T241 cells were transfected with empty vector or vector containing human PDGF-B cDNA expressed by a fused CMV-1E chicken $\beta$-actin promoter (kindly provided by Andras Nagy, Mount Sinai Hospital Research Institute, Toronto, Ontario, Canada), resulting in T241-B cells. Neomycin resistance and lacZ expression were used as selection markers of transfected cells. Selected clones were trypsinized, washed, resuspended in PBS, and injected subcutaneously onto the backs of 6- to 8week-old C57BL6 $(n=6)$ or $p d g f-b^{\text {ret } / \text { ret }}(n=3)$ mice. Each injection of T241 or T241-B cells contained $1 \times 10^{6}$ cells, whereas T241/MEF mixtures contained a total of $1 \times 10^{6}$ cells in a 1:9 ratio. Proliferation studies were performed by seeding $1 \times 10^{4}$ cells $/ \mathrm{cm}^{2}$ (day 0 ), followed by cell counting on days $1,2,4$, and 7 .

The sizes of T241 tumors $(n=10)$ and T241-B tumors $(n=10)$ were estimated by measuring tumor diameter on days $6,9,11$, and 13 after subcutaneous injection of $5 \times 10^{5}$ cells into 7 -week-old C57BL6 females. Tumor volumes were subsequently calculated using the formula $V=\pi \times a^{2} \times b \times 6^{-1}$, where $a$ and $b$ represent the shortest and longest diameter of the tumor, respectively.

Analysis of $m R N A$ and protein expression. PDGF-B expression by T241-B cells was confirmed by Northern blot analysis on confluent cell cultures. Total cellular RNA was isolated with an RNeasy Mini Kit (QIAGEN Inc., Valencia, California, USA), and $10 \mu \mathrm{g}$ RNA was size-fractionated on a denaturing gel and transferred to a BrightStar-Plus membrane using a NorthernMax blotting kit (Ambion Inc., Austin, Texas, USA). Hybridization with ${ }^{32} \mathrm{P}$-labeled cDNA probes for human PDGF-B 
and mouse GAPDH was performed according to the instructions of the manufacturer (Ambion Inc.) and standard protocols.

Quantification of PDGF-B protein levels accumulating in the conditioned medium of confluent $\mathrm{T} 421-\mathrm{B}$ cell cultures was made using the Quantikine PDGF-BB immunoassay kit (R\&D Systems Inc., Minneapolis, Minnesota, USA). Briefly, the medium was conditioned for 2 days and then processed according to the manufacturer's protocol. Optical density was measured using a Vmax microplate reader from Molecular Devices Corp. (Sunnyvale, California, USA). All measurements were repeated and made with replicates. Data were processed using SoftMax 2.35 (Molecular Devices Ltd., Wokingham, United Kingdom).

Immunohistochemistry and in situ hybridization. Tissues were handled as described $(13,32)$. Briefly, tumors were isolated 12-14 days after transplantation and fixed in $4 \%$ paraformaldehyde (PFA) for immunohistochemistry or $0.4 \%$ PFA to enable detection of lacZ expression. Antibody staining was performed on floating sections $40-100 \mu \mathrm{m}$ thick or on $14-\mu \mathrm{M}$ sections on slides. Floating sections were stained for endothelial cells and mounted on glass slides with Mowiol/DABCO (SigmaAldrich, St. Louis, Missouri, USA) mounting medium supplemented with the antibleaching agent DABCO (Sigma-Aldrich) or processed for double and triple labeling. Antibodies used as markers for endothelium were rat anti-Pecam-1 (BD Pharmingen, San Diego, California, USA) and rat anti-endomucin (a kind gift from Dietmar Vestweber, University of Münster, Münster, Germany). VSMCs and pericytes were labeled with rabbit anti-NG2 (Chemicon International, Temecula, California, USA) and FITC-conjugated mouse anti- $\alpha$-smooth muscle actin (SMA) (Sigma-Aldrich). Secondary antibodies conjugated with the appropriate fluorochrome (Alexa 488, 568, or 633; Molecular Probes Inc., Eugene, Oregon, USA) were used. Mounted tumor sections were analyzed by conventional light and fluorescence microscopy using a Nikon E1000 microscope equipped with a digital camera (Coolpix 990; Nikon Inc., Melville, New York, USA) and by confocal laser scanning microscopy using a Leica TCS NT confocal microscope (Leica Microsystems Inc., Deerfield, Illinois, USA). Digital images were processed using Adobe Photoshop 6.0 (Adobe Systems Inc., Mountain View, California, USA).

Nonradioactive in situ hybridization was performed as previously described on frozen sections fixed in $4 \%$ PFA $(20,32)$. RNA probes were transcribed from cDNA fragments coding for the murine and human PDGF-B. Sections were postfixed in 4\% PFA after hybridization and processed as above for immunohistochemical staining of the endothelium.

Quantifications. Vessel density, volume, and length were measured as described (33) using a $1 \mathrm{~mm}^{2}$ point-counting grid covering an area of $0.0625 \mathrm{~mm}^{2}$ at $\times 400$ magnification. These parameters were estimated by counting the number of vessel profiles, test points hitting vessel profiles, and number of intersections with vessel profiles in one line intercept grid. Vessel density is expressed as the number of vessel profiles per $\mathrm{mm}^{2}$. Thirty fields per section and at least two tissue sections were counted from T241 grown on WT mice $(n=7)$, T241 on $p d g f-b^{\text {ret } / \text { ret }}$ mice $(n=5)$, T241-B on WT mice $(n=6)$, and T241-B on $p d g f-b^{\text {ret/ret }}$ mice $(n=3)$. Approximation of the recruited MEF cells in tumors from T241/MEF mixtures was achieved by, in addition to the above-mentioned parameters, scoring lac $\mathrm{Z}^{+}$cells in 10-20 fields of six sections from each tumor type.

Vessel diameters were calculated by measuring at least 100 vessel profiles from six sections of each tumor type (T241 on WT, $n=3$; T241 on $p d g f-b^{\mathrm{ret} / \mathrm{ret}}, n=3$; T241-B on WT, $n=3$; and T241-B on $p d g f-b^{\text {ret } / \text { ret }}$ mice, $\left.n=4\right)$ using Easy Images Measurement 2.1 (Bergström Instruments AB, Solna, Sweden).

Pericyte coverage was measured by calculating the area of overlap of NG2 and Pecam-1 staining. Pericyte density was quantified by relating the NG2-stained area to the Pecam-1-stained area. The actual area of the NG2, Pecam-1, and double-positive stainings were quantified using OpenLab 2.0.7 software (Improvision, Coventry, United Kingdom) from the more than 30 pictures taken from at least five sections ( $14 \mu \mathrm{m}$ thick) of each tumor.

Pericyte detachment was analyzed using OpenLab 2.0.7 software by measuring the shortest distance between the Pecam-1 staining and the most distal point of NG2 staining for individual pericytes and the nearest vessel of confocal images. Between 60 and 100 measurement points were obtained from confocal images of a minimum of four sections of each tumor.

Statistical analysis. All results are expressed as mean \pm SD. Differences between experimental groups were analyzed by Student's $t$ test using two-tailed distribution and the two-sample unequal variance. $P<0.05$ was considered statistically significant.

\section{Results}

Vascular networks developing in different types of tumors vary considerably at different locations in the host and within the same tumor $(8,13,34)$. In general, this complicates the extrapolation of results from one tumor model to another. Here we address the mechanism of tumor vessel pericyte recruitment using a single model, the fibrosarcoma (T241) tumor transplanted subcutaneously, in situations where the host, the tumor cells, and exogenously added pericytes were independently genetically manipulated. T241 tumor vessels normally recruit a significant number of pericytes, although the densities achieved are low in comparison with surrounding normal vessels (13). In addition to being sparse, the pericytes recruited to T241 tumor vessels show an abnormal organization (13).

Deletion of the PDGF-B retention motif leads to decreased mural cell recruitment and increased tumor vessel diameter. Complete inactivation of the $p d g f-b$ gene in mice is embryonic lethal (35). To allow genetic analysis of PDGF-B function in the adult, we have introduced conditional and partially inactivating mutations into the 
$p d g f-b$ gene $(24,36)$. Similar to certain isoforms of other members of the PDGF/VEGF superfamily such as VEGF-A, VEGF-B, placenta growth factor (PlGF), and PDGF-A, the PDGF-B protein contains a conserved C-terminal basic sequence (37) mediating binding within the cell (38), or to proteoglycans at the cell surface and in the ECM (39-42). A similar motif in the VEGF-A protein is necessary for the formation of proper extracellular VEGF-A gradient, which in turn is required for correct angiogenic sprouting and branching morphogenesis (43-45).

We have recently generated mice carrying a knock-in mutation in the $p d g f-b$ gene, leading to C-terminal truncation of the PDGF-B retention motif in the endogenous PDGF-B protein (36). Homozygous carriers of the $p d g f b^{\text {ret }}$ allele $\left(p d g f-b^{\text {ret } / \mathrm{ret}}\right.$ mice) survive into adulthood, but show impaired pericyte recruitment in many vascular beds, most notably in the kidney and the retina (36). The postnatal survival of $p d g f-b^{\text {ret } / \text { ret }}$ mice allowed tumor transplantation experiments and analysis of the importance of cell/matrix-bound PDGF-B in tumor angiogenesis. We transplanted T241 cells into $p d g f-b^{\text {ret/ret }}$ mice in a C57BL6 congenic background. Tumors grown on WT and $p d g f-b^{\text {ret/ret }}$ mice reached similar sizes 12 days after transplantation; however, the tumors grown on $p d g f-b^{\text {ret/ret }}$ hosts were consistently more hemorrhagic at the time of isolation (data not shown). Tumor sections were stained for endothelium (Pecam-1) and mural cells (NG2 or SMA). The vasculature of tumors grown on $p d g f-b^{\text {ret/ret }}$ mice had an increased proportion of large and dilated vessels compared with tumors grown on WT mice (Figure 1, a and b, and Figure 2, a and b). Vessels in the normal tissue surrounding the tumor had a normal appearance both in size and perivascular cell association (Figure 1, c and d). Quantification showed that the mean section diameter of tumor vessels in $p d g f-b^{\text {ret } / \text { ret }}$ mice was approximately twofold greater than that in WT mice (Figure 2, a and b), resulting in a threefold higher total vessel volume relative to the tumor volume $(0.08 \pm 0.003$ vs. $0.03 \pm 0.007, P<0.05)$. The vessel density was not significantly different between tumors grown on WT and pdgf-bet/ret mice (data not shown).

Tumor pericytes express NG2 (high-molecular-weight melanoma-associated antigen) $(7,8)$ in addition to SMA $(11,13)$ and desmin $(34)$. The expression of these markers overlaps extensively, although some tumors appear to have a larger proportion of pericytes expressing NG2 than SMA $(7,8)$. In T241 tumors grown on WT C57BL6 mice, the vessel profiles present on tumor tissue sections showed irregular coverage of NG2-positive cells, varying from dense coats completely covering the abluminal endothelial surface (100\% coverage) to naked stretches of endothelium ( $0 \%$ coverage) (Figure 1a and Figure 2, c-e). The majority of the tumor vessel pericytes were positive for both NG2 and SMA, but rare NG2positive, SMA-negative pericytes could also be identified (data not shown). The NG2-positive cells were invariably tightly associated with the abluminal endothelial surface in T241 tumors grown on WT mice (Figure 1e).
The vessels of tumors grown on $p d g f b^{\text {ret/ret }}$ mice showed a substantial reduction in the density of NG2-positive cells (Figure 1b and Figure 2, c and f). Mural cell coverage and density were quantified from calculations of NG2-, Pecam-1-, and double-stained areas as illustrated in Figure 2c. More than half of the vessel profiles lacked contact with NG2- and SMA-positive cells (Figure 2, $\mathrm{d}$ and e). Moreover, the NG2-positive cells that were found in these tumors were not as tightly associated with the abluminal endothelial surface as were NG2-positive cells in the tumors grown on WT mice (Figure 1, f and g, and Figure 2, g-i). Regularly, parts of these cells extended away from the vessel, and complete detachment from the abluminal surface of the endothelium was often observed (Figure 1, f and g, arrows). Quantification of the detachment (Figure $2 \mathrm{~g}$ ) revealed an average sevenfold increase in the distance between the endothelium and the most distal parts of the pericytes in $p d g f-b^{\text {ret/ret }}$ mice compared with WT mice. Approximately $4 \%$ of the pericytes extended more than $10 \mu \mathrm{m}$ away from the endothelium in WT mice, compared with more than $80 \%$ in $p d f f-b^{\mathrm{ret} / \mathrm{ret}}$ mice.

During development, $p d g f-b^{\text {ret/ret }}$ mice express approximately threefold-reduced levels of PDGF-B mRNA (36).
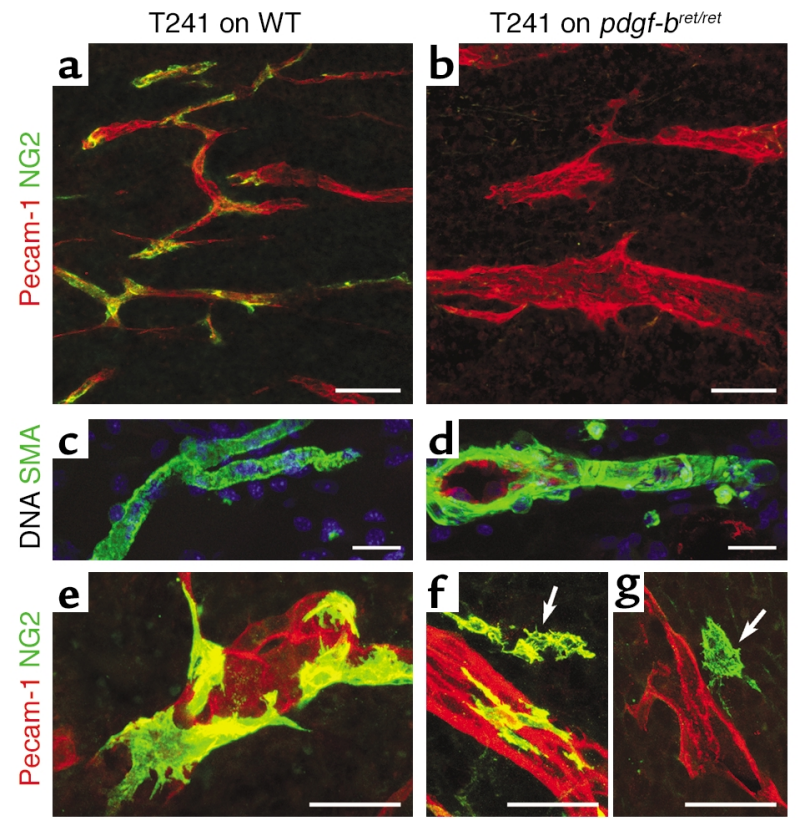

Figure 1

Reduced pericyte recruitment and dilated vessels in tumors transplanted into $p d f f-b^{\text {ret/ret }}$ mice. Double staining of endothelium (Pecam1 , red) and pericytes/VSMCs (SMA or NG2, green) in the vasculature of tumors and surrounding normal tissue of WT and $p d g-b^{\text {ret } / \text { ret }}$ mice. Recruitment of pericytes to tumor vessels was higher in WT (a) than in $p d g f-b^{\text {ret/ret }}$ mice (b), and vessels in tumors grown on $p d g f-b^{\text {ret } / \text { ret }}$ mice were morphologically abnormal and significantly dilated. Vessels in the surrounding dermal tissue show continuous coverage and circular arrangement of mural cells in both WT (c) and pdgf- $b^{\text {ret }}$ /ret mice (d). NG2 staining of the pericytes demonstrated their close association with the endothelium in tumors in WT mice (e), whereas they were partially or completely detached from the tumor endothelium in $p d g f-b^{\text {ret/ret }}$ mice (f and $\mathbf{g}$, arrows). Bars: $50 \mu \mathrm{m}$. 

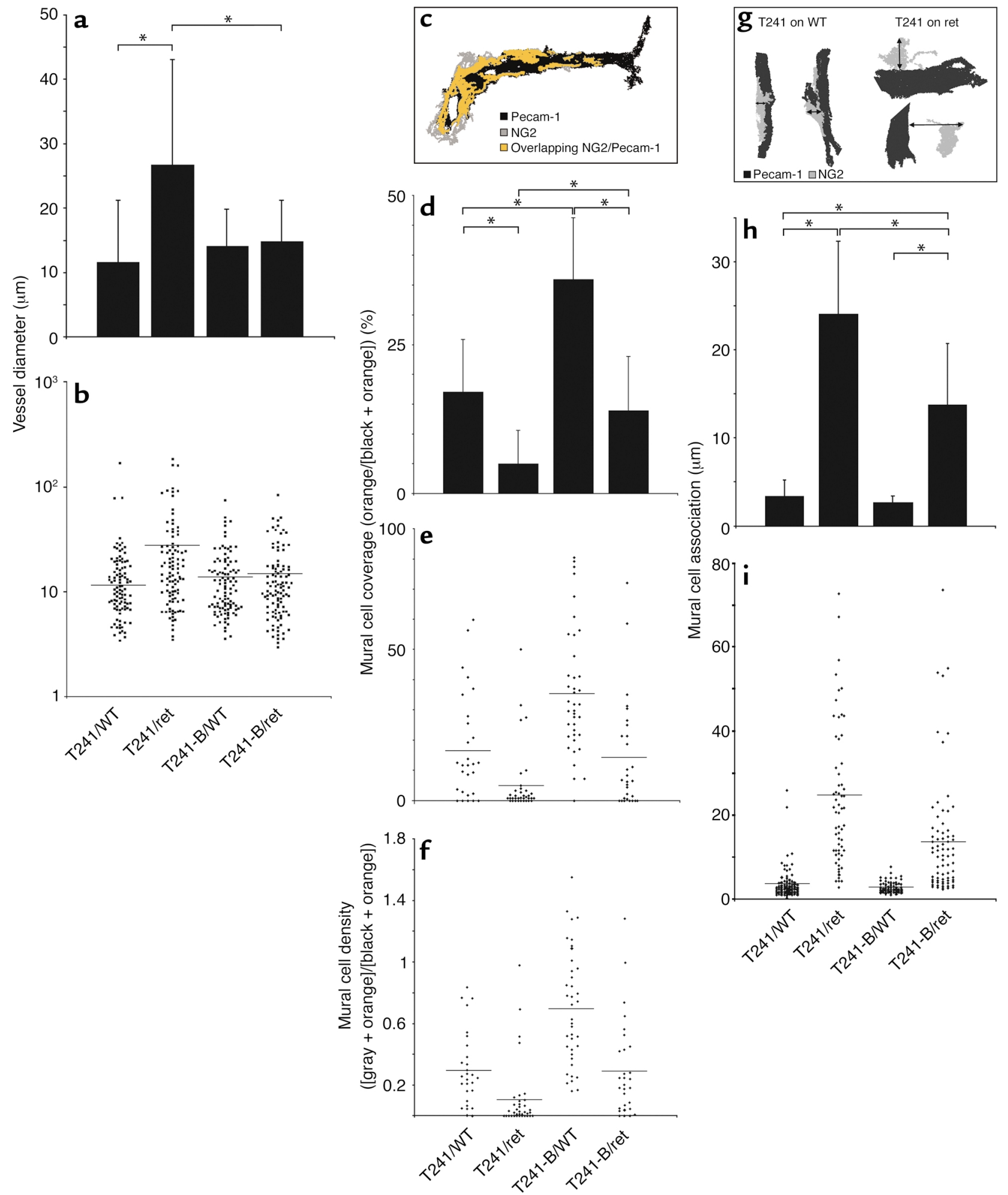

\section{Figure 2}

Quantitative analyses of tumor vessels in T241 tumors grown on WT C57BL6 mice (T241/WT), T241 tumors grown on pdgf-bret/ret mice (T241/ret), T241-B tumors grown on WT C57BL6 mice (T241-B/WT), and T241-B tumors grown on pdff-bet/ret mice (T241-B/ret). (a) Mean vessel diameter. T241/WT, $11.7 \pm 9$; T241/ret, $26.8 \pm 16$; T241-B/WT, $14.5 \pm 5.5$; T241-B/ret, $14.9 \pm 6.5 \mu \mathrm{m}$. (b) Scatter plot of data in a. (c-f) Analysis of pericyte density and coverage. (c) Example of collected data. (d) Pericyte coverage illustrated as NG2-Pecam-1 overlapping area, expressed as a percentage of total Pecam-1-stained area. (e) Scatter plot of the data in d. (f) Pericyte density illustrated as NG2 area as a percentage of total Pecam-1 area. (g-i) Pericyte "detachment" from the endothelial cells expressed as the distance (in $\mu \mathrm{m}$ ) from the endothelium to the most distal part of a pericyte. (g) Example of collected data. (h) Mean distance $(\mu \mathrm{m})$ of mural cell association with tumor endothelium. (i) Scatter plot of individual NG2-positive pericytes. ${ }^{*} P<0.05$. 
T241 on WT

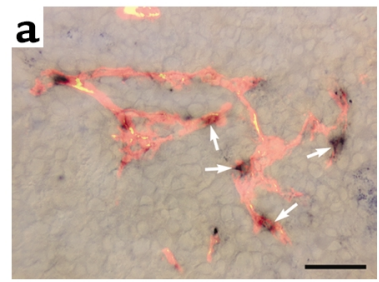

c

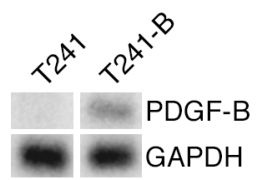

T241 on pdgf- $b^{\text {ret/ret }}$

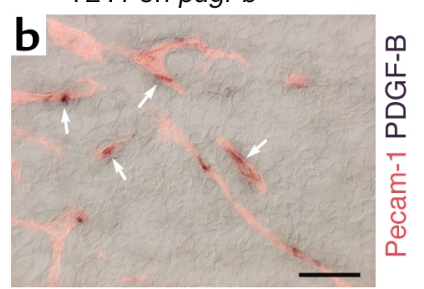

T241 on WT

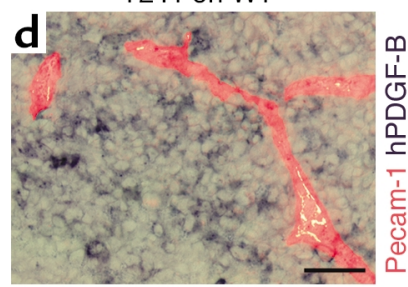

\section{Figure 3}

PDGF-B expression analysis. In situ hybridization revealed a similar pattern of PDGF-B expression (dark blue, arrows) by the endothelium (Pecam-1, red) of T241 tumors grown on (a) WT C57BL6 mice and (b) $p d g f-b^{\text {ret/ret }}$ mice. PDGF-B-transfected T241 cells (T241-B cells) expressed PDGF-B both in vitro, as shown by Northern blot analysis (c), and in vivo, as shown by in situ hybridization of sections of T241-B tumors double stained for vessels (Pecam-1, red) (d). GAPDH hybridization was used to control for RNA loading. hPDGF-B, human PDGF-B. Bars: $50 \mu \mathrm{m}$.

In accordance with these observations, the endothelium of T241 tumors grown on $p d g f-b^{\text {ret/ret }}$ mice expressed PDGF-B mRNA detectable by in situ hybridization, but the signal was reduced compared with the tumor endothelium in WT mice (Figure 3, $a$ and b, arrows). In agreement with previously published data (13), the T241 cells did not express PDGF-B in vitro (Figure 3c) or in tumors in vivo, irrespective of host genotype (Figure 3 , $a$ and $b$ ). Deletion of the retention motif generates a PDGF-B protein that is less cell-associated than WT PDGF-B $(36,39,40)$. We have not been able to immunolocalize endogenously expressed and secreted PDGF-B protein in tissues, and therefore we are not able to test whether the $p d g f-b^{\text {ret }}$ mutation leads to altered extracellular distribution of the endogenous PDGF-B protein in vivo in $p d g f-b^{\text {ret} / \mathrm{ret}}$ mice. However, the distribution in the dermis of recombinant WT and C-terminally truncated PDGF-B expressed by transplanted keratinocytes suggests that secreted WT PDGF$B$ protein becomes bound in the ECM surrounding the producer cells, whereas the mutant PDGF-B diffuses more freely in the tissue (42). Moreover, using endothelioma cultures established from WT and $p d g f-b^{\text {ret } / \text { ret }}$ mice, we have shown that the endogenous PDGF-Bret protein more readily accumulates in the cell culture medium than does the corresponding WT PDGF-B protein (36). Together, these results suggest that retention of PDGF-B in the close vicinity of the endothelial cells is critical for tumor pericyte recruitment. In addition, our data show that PDGF-B also has a role in keeping recruited pericytes tightly associated with the tumor vessel endothelium and that the retention motif is critical for this function.

PDGF-B expression by T241 tumor cells increases mural cell recruitment. The data above suggest that tumor vessel-derived PDGF-B has a critical role in pericyte recruitment in the $\mathrm{T} 241$ model. In contrast to the T241 cells, many tumor cells express PDGF-B, which prompted us to analyze whether tumor cell-derived PDGF-B could promote pericyte recruitment to tumor vessels as well. To address this, we transfected T241
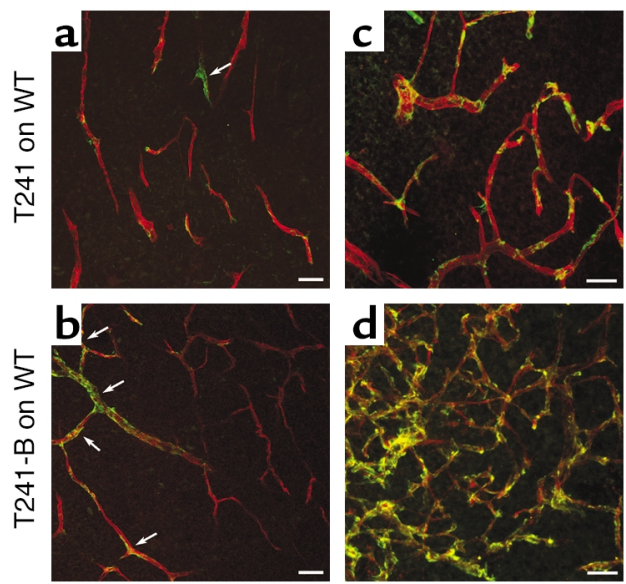

\section{NG2 Endomucin}
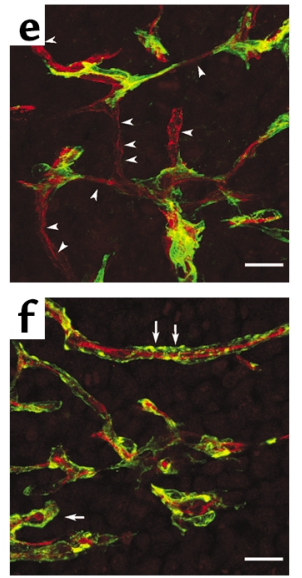
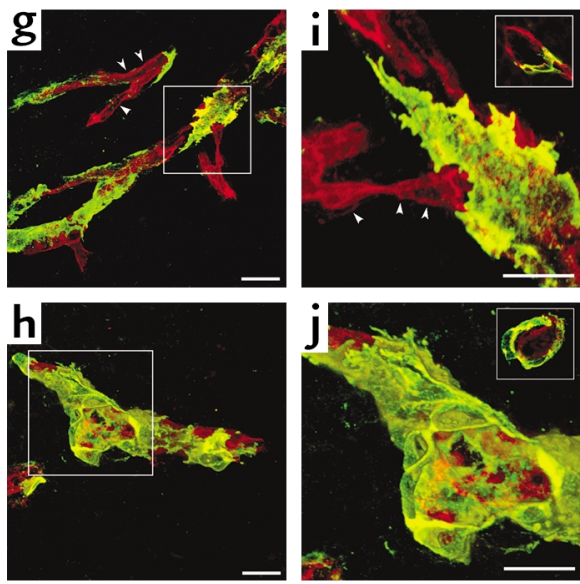

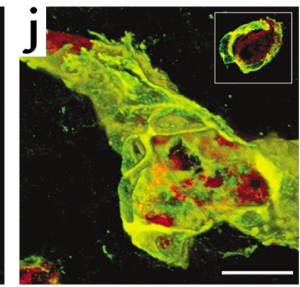

Figure 4

Tumor cell-derived PDGF-B increases pericyte recruitment to tumor vessels. Tumor vessel endothelium is visualized by staining for endomucin (red) and pericytes by staining for NG2 (green). Low magnification shows the increased density of mural cells in central (a and $\mathbf{b}$, arrows) and peripheral areas (c and d) of T241 and T241-B tumors, respectively, transplanted into WT C57BL6 mice. In parental T241 tumors, a significant proportion of the abluminal endothelial surface lacks associated pericytes (e, $\mathbf{g}$, and $\mathbf{i}$, arrowheads). In contrast, an almost continuous layer of pericytes is seen in T241-B tumors (f $\mathbf{f}$, arrows). Analysis at higher magnification reveals that the pericytes in T241-B tumors tend to circular arrangements $(\mathbf{h})$, whereas the pericytes in parental T241 tumors show a more longitudinal arrangement $(\mathbf{g})$. $\mathbf{i}$ and $\mathbf{j}$ show closeups of the insets in $\mathbf{g}$ and $\mathbf{h}$. Compare also mural cell encircling of cross-sectioned vessels (insets in $\mathbf{i}$ and $\mathbf{j})$. Bars: $50 \mu \mathrm{m}(\mathbf{a}-\mathbf{f})$ and $20 \mu \mathrm{m}(\mathbf{g}-\mathbf{j})$. 


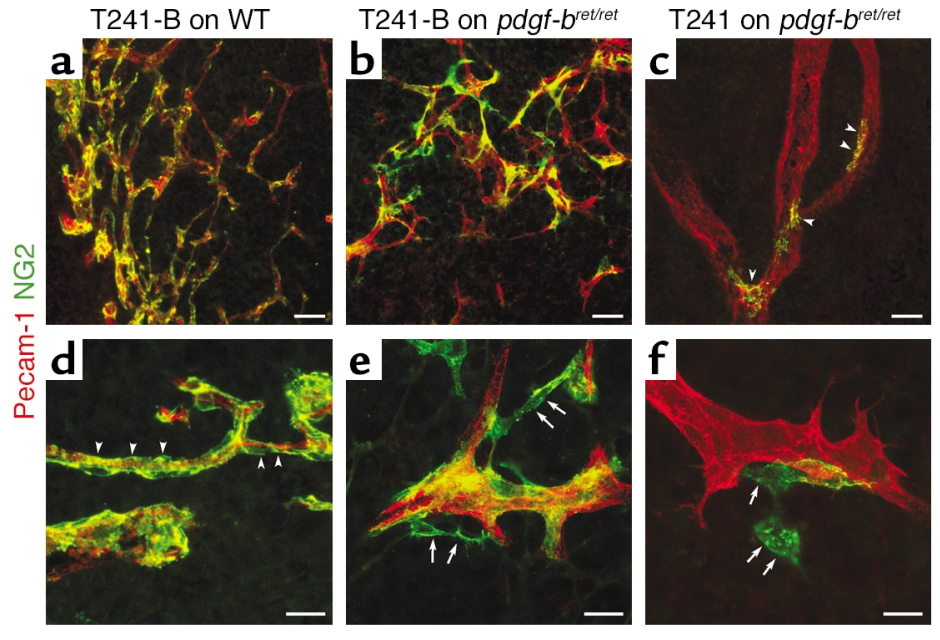

Figure 5

Increased pericyte number but failure of proper pericyte investment in T241-B tumors transplanted into $p d g-b^{\text {ret/ret }}$ mice. Pecam-1 (red) and NG2 (green) double staining of endothelium and pericytes, respectively, reveals that PDGF-B produced by the T241-B tumors can partially rescue the defective pericyte number in $p d g f-b^{\text {ret/ret }}$ mice (b and $\mathbf{c}$, arrowheads). The pericyte density was, however, lower than that obtained after transplantation of T241-B tumors into WT mice (a). In spite of the partial rescue of pericyte number by T241-derived PDGF-B, the proper arrangement of pericytes in the vessel wall was dependent on expression of WT PDGF-B protein by the host endothelium. Note the partial or complete detachment of a majority of the pericytes in the $p d g f-b^{\text {ret }} /$ ret mice, irrespective of whether the T241 cells express PDGF-B or not (b, and $\mathbf{e}$ and $\mathbf{f}$, arrows), compared with T241-B tumors in WT mice (d, arrowheads). Bars: 50 $\mu \mathrm{m}(\mathbf{a}-\mathbf{c})$ and $20 \mu \mathrm{m}(\mathbf{d}-\mathbf{f})$.

cells with an expression vector for human PDGF-B (resulting in T241-B cells). PDGF-B expression was confirmed in selected subclones by Northern blot analysis and RT-PCR (Figure 3c and data not shown), and approximately $10 \mathrm{pg} / \mathrm{ml}$ of PDGF-B protein was measured in the conditioned medium of T241-B cultures by a human PDGF-B immunoassay (data not shown). In vivo expression of human PDGF-B by transfected T241 tumors was confirmed by in situ hybridization (Figure 3d). In vitro proliferation of the T241-B cells was slightly reduced (approximately 20\%) in comparison with the maternal T241 cells, probably a result of clonal variability (data not shown). The in vivo growth rate of T241-B tumors was also slightly reduced compared with maternal T241 tumors; however, this difference was not statistically significant in ten tumors of each type (data not shown).

T241-B cells gave rise to tumors with a significantly increased density of mural cells in the tumor vessels compared with the maternal T241 cells (Figure $2 \mathrm{f}$ and Figure 4). This was true both in the central regions of the tumor, where pericyte density is normally sparse (Figure $4 \mathrm{a}$ and $\mathrm{b}$, arrows), and at the periphery, where pericyte coating is normally more dense (Figure 4, c and $\mathrm{d}$ ). In T241 tumors, only a relatively small proportion $(17 \%)$ of the abluminal endothelial surface was covered by mural cells, leaving most of the abluminal surface naked even in the most pericyte-dense areas
(Figure 4, e, g, and i, arrowheads). In T241-B tumors, the mural cell coverage was significantly increased (36\%) (Figure 2, d and e), and sites were found in the periphery of the tumor where pericytes almost completely covered the abluminal endothelial surface (Figure 4, f, $h$, and $j$, arrows; see also insets in $i$ and $j$ for a view of a typical vessel cross section). This high degree of coverage was never observed in tumors arising from the maternal T241 cells.

Also, T241-B cells transplanted to $p d g f-b^{\text {ret/ret }}$ mice recruited significantly more pericytes than the T241 cells, leading to a normalized pericyte density in comparison with T241 tumors grown on WT mice (Figure 2f). Thus, tumor-derived PDGF-B seems capable of compensating for the loss of endothelial PDGF-B retention. The pericyte coverage was also significantly increased, but not fully compensated for in comparison with T241 on WT mice (Figure 2, $\mathrm{d}$ and e). The discrepancy between the degree of compensation of pericyte density and coverage is explained by the pericyteendothelium association; similar to the T241 tumors on $p d g f-b^{\text {ret} / \text { ret }}$ mice, the T241-B tumors on $p d g f-b^{\text {ret/ret }}$ mice showed partial detachment of the mural cells (Figure 2, g-i, and Figure 5, $b$, e, and $f$, arrows). Thus, an endothelial source of WT PDGF-B protein appears to be required for the establishment of a tight association between the endothelial cells and mural cells in tumor vessels, and this source cannot be compensated for by tumor-derived PDGF-B.

Pericyte recruitment to tumor vessels requires $P D G F-R \beta$. In developmental angiogenesis, pericyte recruitment is mediated by PDGF-B binding to PDGF-R $\beta(20,21)$. Since PDGF-R $\beta$ knockouts are embryonic lethal (46), we tested the importance of PDGF-R $\beta$ for tumor pericyte recruitment using cell transplantation. We recently showed that the vasculature within the T241 tumor is capable of efficiently recruiting ectopic pericytes from coinjected cultures of MEFs (13). To determine whether PDGF-R $\beta$ expression by MEFs is required in this process, tumors were grown from a mixture of T241 cells and MEF cells isolated from WT or PDGF-R $\beta$ null embryos harboring the transgenic marker XlacZ4, which is expressed in the cell nuclei of pericytes and VSMCs $(13,23,47)$. Although PDGF-R $\beta$-negative MEF cells grew more slowly in culture than the corresponding WT cells (data not shown), a similar proportion (approximately 10\%) of XlacZ4-positive cells was observed in both types of MEF cultures (Figure 6, a and b). This was not unexpected since the MEFs are derived from embryonic day 12.5 embryos, which in contrast to late-stage embryos generally have normal numbers of mural cells in the absence of PDGF-B or PDGF-R $\beta(21)$. The MEF cultures were prelabeled with a fluorescent cell membrane marker, Pkh26, to allow tracing within the tumor. In tumors containing WT MEFs, a propor- 

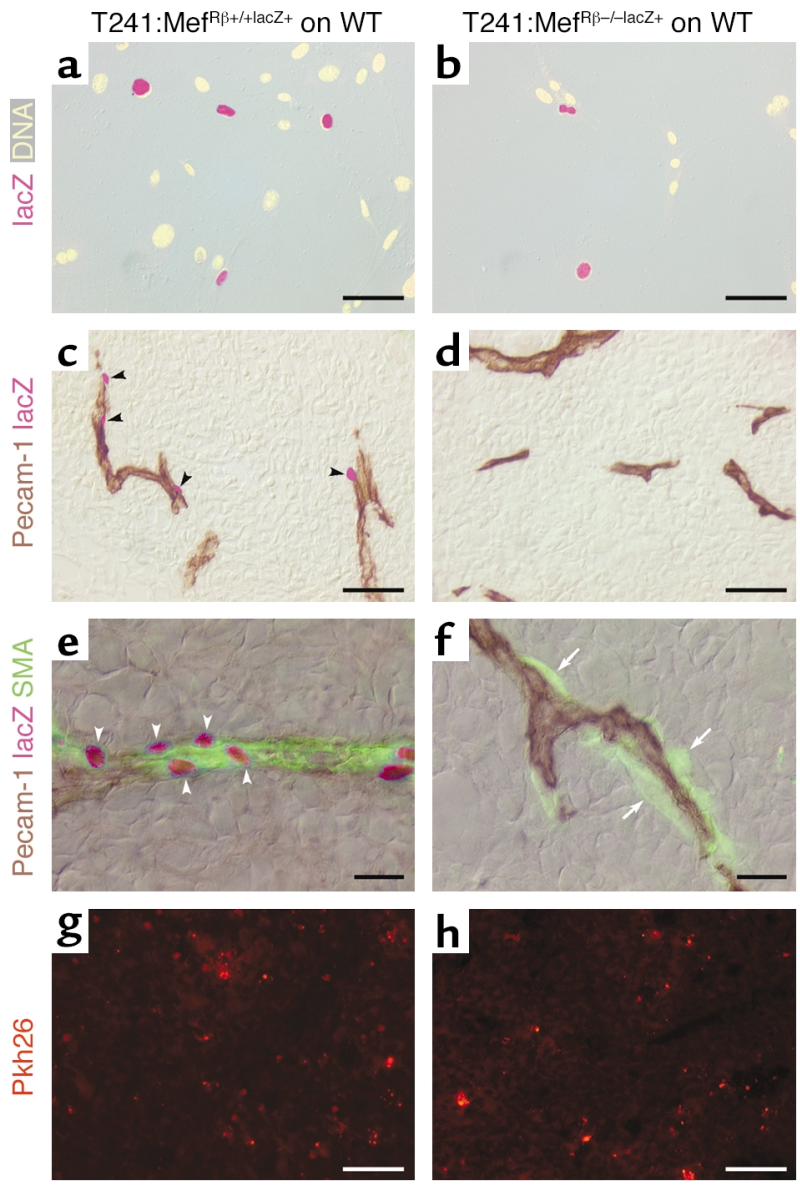

tion of the vessel profiles showed tightly associated lacZ-positive nuclei (Figure 6c, arrowheads) in cells coexpressing SMA (Figure 6e, arrowheads). In contrast, no vessel-associated lacZ-positive nuclei were found in tumors transplanted together with PDGF-R $\beta$-deficient MEFs (Figure 6d). While these tumors failed to recruit the exogenously added MEFs, they showed recruitment of endogenous SMA-positive pericytes (Figure 6f, arrows). Pkh26 labeling demonstrated a similar number and distribution of PDGF-R $\beta$-negative and WT MEFs within the tumor, indicating that the lack of PDGF-R $\beta$ negative lacZ-positive cells in the tumors is not a result of uneven spreading or generally reduced survival of the transplanted PDGF-R $\beta$-negative MEFs (Figure 6, g and h). These results suggest that pericyte recruitment to T241 tumor vessels requires PDGF-R $\beta$ expression by the pericytes or their progenitors.

Loss of PDGF-B retention does not block recruitment of exogenous mural cells but impairs their integration in the vessel wall. We next injected WT MEFs derived from XlacZ4 transgenic embryos together with T241 tumor cells into $p d g f-b^{\text {ret/ret }}$ mice. In the resulting tumors, XlacZ4-positive cells were found at the same frequency as in tumors transplanted into WT mice $(2.3 \pm 1.2$ cells $/ \mathrm{mm}$ of vessel length in WT mice compared with $3.8 \pm 1.8$ cells $/ \mathrm{mm}$ in $p d g f-b^{\text {ret } / \text { ret }}$ mice) (Figure 7 , a and $b$, arrowheads). This suggests that PDGF-B retention is

\section{Figure 6}

Exogenous PDGF-R $\beta$-deficient pericytes are not recruited by tumor vessels. MEFs were isolated from XlacZ4-positive WT or PDGF-R $\beta$ negative embryonic day 12.5 embryos and cultured in vitro (a and b). These cultures contained similar proportions of XlacZ4-positive cells $(\sim 10 \%)$. T241 cells mixed with MEFs at a 1:9 ratio were injected subcutaneously onto the backs of WT mice. Cells expressing lacZ (pink) were found closely associated with endothelial cells (Pecam-1, brown) in tumors mixed with PDGF-R $\beta$-positive MEF cells (c, arrows) but not in tumors mixed with PDGF-R $\beta$-negative MEF cells (d). Triple staining shows that the recruited lacZ-positive MEF cells (e, arrowheads) also express SMA (green) and that the lacZ/SMA double-positive cells are tightly associated with the endothelium (brown) (e). Vessels in tumors mixed with PDGF-R $\beta$-negative MEF cells lack exogenous (lacZ-positive) pericytes but recruit endogenous (lacZ-negative, SMA-positive) pericytes, as expected (f, arrows). Prelabeling of MEF cultures with Pkh26 dye indicates the presence and similar distribution of PDGF-R $\beta$-positive and -negative MEF cells within the tumors ( $\mathbf{g}$ and $\mathbf{h}$ ). Bars: $100 \mu \mathrm{m}$ ( $\mathbf{a}$ and $\mathbf{b}), 50 \mu \mathrm{m}(\mathbf{c}, \mathbf{d}, \mathbf{g}$, and $\mathbf{h}$ ), and $20 \mu \mathrm{m}$ (e and $\mathbf{f}$ ).

not absolutely required for attraction of pericytes to the vessels when pericytes are available for such recruitment. Previous analysis of embryonic angiogenesis has demonstrated that PDGF-B/PDGF-R $\beta$ signaling stimulates pericyte proliferation (21). This may explain the discrepancy between the recruitment of endogenous pericytes (reduced) and the attraction of exogenously added pericytes (normal) in $p d g f-b^{r e t / r e t}$ mice; the former requires proliferation because the source of endogenous pericytes available for recruitment is limited (13). However, whereas the exogenously added pericytes were efficiently attracted to the tumor vessels, they showed a looser attachment to the endothelium in $p d g f-b^{r e t / r e t}$ mice than in WT mice (Figure $7, c$ and $d$

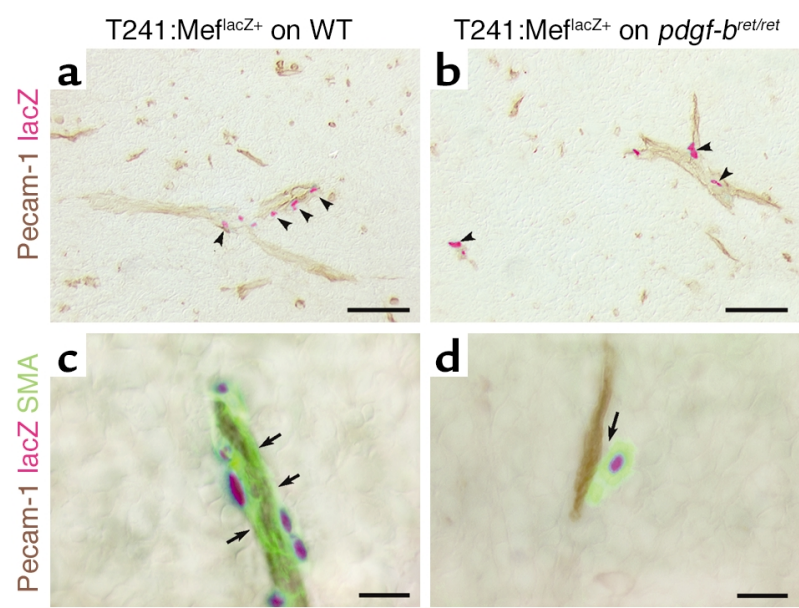

\section{Figure 7}

Exogenous pericyte recruitment in $p d g f-b^{\text {ret/ret }}$ mice. Exogenous XlacZ4-positive MEF cells became associated with tumor vessels in both WT (a, arrowheads) and pdgf- $b^{\text {ret/ret }}$ mice (b, arrowheads). Double staining for lacZ (pink) and SMA (green) revealed defective association between the exogenously recruited pericytes and the endothelium in $p d g f-b^{\text {ret/ret }}$ tumors (d, arrow) but not in WT tumors (c, arrows). Bars: $100 \mu \mathrm{m}$ (a and b) and $20 \mu \mathrm{m}$ (c and d). 


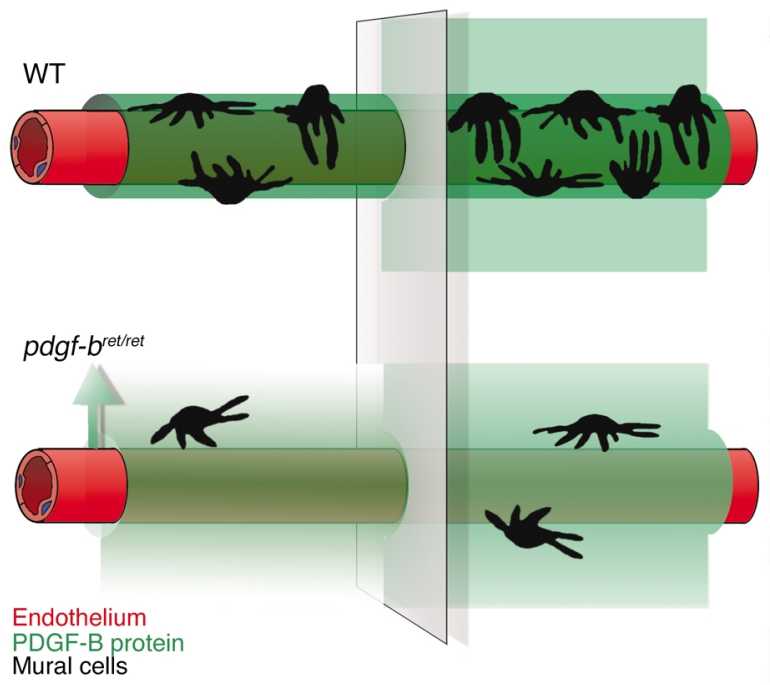

Figure 8

Model for perivascular PDGF-B protein distribution in T241 tumors and its effect on tumor vessel pericytes. WT PDGF-B protein has affinity for heparan-sulfate proteoglycans and other ECM molecules. Its expression by the endothelium is therefore expected to give rise to a depot or steep gradient of PDGF-B in the periendothelial compartment (upper left). This promotes a certain amount of pericyte recruitment, and moreover, the recruited pericytes become intimately associated with the abluminal surface of the endothelium. Additional PDGF-B protein secreted by the tumor cells (upper right) leads to additional pericyte recruitment, with retained association to the endothelium. In $p d g f-b^{\text {ret/ret }}$ mice, the PDGF-B protein lacks the retention motif, and is therefore more freely diffusible following its release from the endothelial cells (lower left). The lower concentration or shallower gradient in the periendothelial compartment leads to reduced pericyte recruitment and defective investment of the pericytes in the microvessel wall. Additional PDGF-B protein secreted by the tumor cells (lower right) promotes the recruitment of higher numbers of pericytes, which remain abnormally associated with the endothelium.

arrows), similar to the situation observed for the endogenous pericytes in tumors grown on $p d g f-b^{\text {ret }}$ /ret mice (Figure 1, $\mathrm{f}$ and $\mathrm{g}$ ).

\section{Discussion}

Using a combination of genetic gain- and loss-of-function approaches, we demonstrate that the PDGF-B/ PDGF-R $\beta$ system has a critical function for pericyte recruitment to tumor vessels. Pericyte recruitment depends on PDGF-B expression by the tumor vessel endothelium, and since tumor pericytes express PDGF-R $\beta$ $(13,26)$, the mode of signaling is short-range paracrine. Additional indications for the importance of this mode of signaling are provided by observations on $p d g f-b^{\text {ret/ret }}$ mice. In these mutants, pericyte recruitment is sufficient to support embryonic and postnatal development and vital organ function (with the exception of the eyes) (36). However, the number of recruited pericytes in $p d g f-b^{\text {ret/ret }}$ mice is lower than normal, and the recruited pericytes do not become tightly integrated in the vessel wall. The lower number of pericytes may be explained by decreased proliferation of the pericytes or their progenitors. This would be expected if the local concentration of PDGF-B is decreased in the periendothelial region as a result of reduced retention of the PDGF-B protein at the endothelial cell surface or in the immediately surrounding ECM. The loose association of pericytes with the vessel wall in $p d g f-b^{\text {ret/ret }}$ mice is intriguing. Since PDGF-B secretion from the tumor cells could rescue the number of recruited pericytes, but not the vessel wall integration defect, it is likely that the pericytes respond specifically to a localized endothelial source of PDGF-B. This response may include directed subcellular localization of adhesion molecules to facilitate endothelium-pericyte adhesion or localized ECM production. The pericyte response to the expected differences in PDGF-B distribution in T241 tumors and $p d g f-b^{\text {ret/ret }}$ mice is schematically illustrated in Figure 8.

It is noteworthy that while T241 tumors normally acquire vessels with tightly associated pericytes, certain other tumors have loosely associated pericytes even when transplanted into WT mice (34). It is possible that these tumors produce matrix molecules or growth factors that trigger pericyte migration away from the vessels by competing with the endothelial source of PDGF-B and/or by providing a pericyte-adhesive matrix. PDGF-B secretion by the tumor cells would be expected to direct pericyte migration away from the vessels if the PDGF-B concentrations become large enough to overwhelm the periendothelial gradient, or depot, of PDGF-B. It is likely that the lack of PDGF-B expression by maternal T241 cells, possibly in combination with other factors, leads to the generation of a relatively normal vasculature in which pericyte investment and association with the vessel wall depends on the endothelial source of PDGF-B. In any case, the T241 model was highly suitable for the purpose of the present study since it generated interpretable results in both gain- and loss-of-function analyses, revealing necessary and sufficient functions of PDGF-B in pericyte recruitment to tumor vessels.

Insight into mechanisms that lead to pericyte recruitment to tumor vessels may have important implications for the development of antiangiogenic strategies to suppress tumor growth. Published reports have indicated that pericyte-covered and non-pericyte-covered vessels are differentially sensitive to VEGF withdrawal and antiangiogenic treatment with IL-12 $(12,15)$. Moreover, the antitumor effect of certain broad-spectrum kinase inhibitors, and of combinations of kinase inhibitors, may be explained in part by an effect on pericytes $(10,26)$. Our own data suggest that pericyte density has significant effects on tumor vessel morphology. Loss of pericytes correlates with vessel dilation and tumor hemorrhaging. The pericyte-deficient tumors

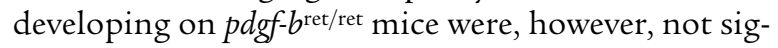
nificantly reduced in size compared with tumors grown on WT mice. Thus, the degree of pericyte deficiency obtained in $p d g f-b^{\text {ret/ret }}$ mice is still sufficient to support tumor growth. In preliminary experiments we have noticed that the tumors grown on $p d g f-b^{\text {ret/ret }}$ mice have 
a changed pattern of expression and higher levels of VEGF-A (A. Abramsson et al., unpublished observations). Future studies will hopefully reveal whether these tumors show increased VEGF dependence and greater sensitivity to antiangiogenic therapy directed to VEGF-A or its signaling pathways.

\section{Acknowledgments}

We would like to thank Magdalena Kegel for her initial work on the establishment and analysis of the T241-B cells and Holger Gerhardt for valuable suggestions on technical issues and helpful comments on the manuscript. This study was supported by grants from the Swedish Cancer Foundation, the Inga-Britt and Arne Lundberg Foundation, the Novo Nordisk Foundation, Stiftelsen Ragnhild and Einar Lundströms Memory, and Konrad and Helfrid Johanssons Research Foundation. A. Abramsson is supported by a fellowship from the Cardiovascular Program of the Swedish Foundation for Strategic Research.

1. Folkman, J. 1971. Tumor angiogenesis: therapeutic implications. N. Engl. J. Med. 285:1182-1186.

2. Yuan, F., et al. 1994. Vascular permeability and microcirculation of gliomas and mammary carcinomas transplanted in rat and mouse cranial windows. Cancer Res. 54:4564-4568.

3. Yuan, F., et al. 1995. Vascular permeability in a human tumor xenograft: molecular size dependence and cutoff size. Cancer Res. 55:3752-3756.

4. Hashizume, H., et al. 2000. Openings between defective endothelial cells explain tumor vessel leakiness. Am. J. Pathol. 156:1363-1380.

5. Hobbs, S.K., et al. 1998. Regulation of transport pathways in tumor vessels: role of tumor type and microenvironment. Proc. Natl. Acad. Sci. U. S. A. 95:4607-4612.

6. Jain, R.K. 1988. Determinants of tumor blood flow: a review. Cancer Res. 48:2641-2658.

7. Schlingemann, R.O., Rietveld, F.J., de Waal, R.M., Ferrone, S., and Ruiter, D.J. 1990. Expression of the high molecular weight melanoma-associated antigen by pericytes during angiogenesis in tumors and in healing wounds. Am. J. Pathol. 136:1393-1405.

8. Schlingemann, R.O., et al. 1991. Differential expression of markers for endothelial cells, pericytes and basal lamina in the microvasculature of tumors and granulation tissue. Am. J. Pathol. 138:1335-1347.

9. Wesseling, P., et al. 1995. Early and extensive contribution of pericytes/ vascular smooth muscle cells to microvascular proliferation in glioblastoma multiforme: an immuno-light and immuno-electron microscopic study. J. Neuropathol. Exp. Neurol. 54:304-310.

10. Shaheen, R., et al. 2001. Tyrosine kinase inhibitors of multiple angiogenic growth factor receptors improves survival in mice bearing colon cancer liver metastases by inhibition of endothelial cell survival mechanisms. Cancer Res. 61:1464-1468.

11. Eberhard, A., et al. 2000. Heterogeneity of angiogenesis and blood vessel maturation in human tumors: implications for antiangiogenic therapies. Cancer Res. 60:1388-1393.

12. Benjamin, L.E., Golijanin, D., Itin, A., Pode, D., and Keshet, E. 1999. Selective ablation of immature blood vessels in established human tumors follows vascular endothelial growth factor withdrawal. J. Clin. Invest. 103:159-165.

13. Abramsson, A., et al. 2002. Analysis of mural cell recruitment to tumor vessels. Circulation. 105:112-117.

14. Folkman, J. 1985. Tumor angiogenesis. Adv. Cancer Res. 43:175-203.

15. Gee, M.S., et al. 2003. Tumor vessel development and maturation impose limits on the effectiveness of anti-vascular therapy. Am. J. Pathol. 162:183-193.

16. Brooks, P.C., Clark, R.A., and Cheresh, D.A. 1994. Requirement of vascular integrin alpha $v$ beta 3 for angiogenesis. Science. 264:569-571.

17. Arap, W., Pasqualini, R., and Rouslahti, E. 1998. Cancer treatment by targeting drug delivery to tumor vasculature in a mouse model. Science. 279:277-280.

18. Tarli, L., et al. 1999. A high-affinity human antibody that targets tumoral blood vessels. Blood. 94:192-198.

19. Sims, D.E. 1986. The pericyte-a review. Tissue Cell. 18:153-174.

20. Lindahl, P., Johansson, B.R., Levéen, P., and Betsholtz, C. 1997. Pericyte loss and microaneurysm formation in PDGF-B-deficient mice. Science. 277:242-245.

21. Hellström, M., Kalén, M., Lindahl, P., Abramsson, A., and Betsholtz, C. 1999. Role of PDGF-B and PDGFR-beta in recruitment of vascular smooth muscle cells and pericytes during embryonic blood vessel formation in the mouse. Development. 126:3047-3055.

22. Hellström, M., et al. 2001. Lack of pericytes leads to endothelial hyperplasia and abnormal vascular morphogenesis. J. Cell. Biol. 153:543-553.

23. Klinghoffer, R.A., Mueting-Nelsen, P.F., Faerman, A., Shani, M., and Soriano, P. 2001. The two PDGF receptors maintain conserved signaling in vivo despite divergent embryological functions. Mol. Cell. 7:343-354.

24. Enge, M., et al. 2002. Endothelium-specific platelet-derived growth factor-B ablation mimics diabetic retinopathy. EMBOJ. 21:4307-4316.

25. Uemura, A., et al. 2002. Recombinant angiopoietin-1 restores higherorder architecture of growing blood vessels in mice in the absence of mural cells. J. Clin. Invest. 110:1619-1628. doi:10.1172/JCI200215621.

26. Bergers, G., Song, S., Meyer-Morse, N., Bergsland, E., and Hanahan, D. 2003. Benefits of targeting both pericytes and endothelial cells in the tumor vasculature with kinase inhibitors. J. Clin. Invest. 111:1287-1295. doi:10.1172/JCI200317929.

27. Guo, P., et al. 2003. Platelet-derived growth factor-B enhances glioma angiogenesis by stimulating vascular endothelial growth factor expression in tumor endothelia and by promoting pericyte recruitment. Am.J. Pathol. 162:1083-1093.

28. Westermark, B., and Heldin, C.-H. 1991. Platelet-derived growth factor in autocrine transformation. Cancer Res. 51:5087-5092.

29. Forsberg, K., Valyi, N.I., Heldin, C.H., Herlyn, M., and Westermark, B. 1993. Platelet-derived growth factor (PDGF) in oncogenesis: development of a vascular connective tissue stroma in xenotransplanted human melanoma producing PDGF-BB. Proc. Natl. Acad. Sci. U. S. A. 90:393-397.

30. Heldin, C.H., and Westermark, B. 1999. Mechanism of action and in vivo role of platelet-derived growth factor. Physiol. Rev. 79:1283-1316.

31. Pietras, K., et al. 2001. Inhibition of platelet-derived growth factor receptors reduces interstitial hypertension and increases transcapillary transport in tumors. Cancer Res. 61:5778-5783.

32. Hogan, B., Beddington, R., Costantini, F., and Lacy, E. 1994. Manipulating the mouse embryo. A laboratory manual. Cold Spring Harbor Laboratory Press. Cold Spring Harbor, New York, USA. 373-375.

33. Gundersen, H.J., et al. 1988. Some new, simple and efficient stereological methods and their use in pathological research and diagnosis. APMIS. 96:379-394.

34. Morikawa, S., et al. 2002. Abnormalities in pericytes on blood vessels and endothelial sprouts in tumors. Am. J. Pathol. 160:985-1000.

35. Levéen, P., et al. 1994. Mice deficient for PDGF B show renal, cardiovascular, and hematological abnormalities. Genes Dev. 8:1875-1887.

36. Lindblom, P., et al. 2003. Endothelial PDGF-B retention is required for proper investment of pericytes in the microvessel wall. Genes Dev. 17:1835-1840.

37. Betsholtz, C., Rorsman, F., Westermark, B., Östman, A., and Heldin, C.-H. 1990. Analogous alternative splicing. Nature. 344:299.

38. Thyberg, J., Östman, A., Bäckström, G., Westermark, B., and Heldin, C.-H. 1990. Localization of platelet-derived growth factor (PDGF) in CHO cells transfected with PDGF A- or B-chain CDNA: retention of PDGF-BB in the endoplasmic reticulum and Golgi complex. J. Cell Sci. 97:219-229.

39. Östman, A., Andersson, M., Betsholtz, C., Westermark, B., and Heldin, C.-H. 1991. Identification of a cell retention signal in the B-chain of PDGF and in the long splice version of the A-chain. Cell Regul. 2:503-512.

40. LaRochelle, W.J., May-Siroff, M., Robbins, K.C., and Aaronson, S.A. 1991. A novel mechanism regulating growth factor association with the cell surface: identification of a PDGF retention domain. Genes Dev. 5:1191-1199.

41. Raines, E.W., and Ross, R. 1992. Compartmentalization of PDGF on extracellular binding sites dependent on exon-6-encoded sequences. J. Cell Biol. 116:533-543.

42. Eming, S.A., Yarmush, M.L., Krueger, G.G., and Morgan, J.R. 1999. Regulation of the spatial organization of mesenchymal connective tissue: effects of cell-associated versus released isoforms of platelet-derived growth factor. Am. J. Pathol. 154:281-289.

43. Stalmans, I., et al. 2002. Arteriolar and venular patterning in retinas of mice selectively expressing VEGF isoforms. J. Clin. Invest. 109:327-336. doi:10.1172/JCI200214362.

44. Ruhrberg, C., et al. 2002. Spatially restricted patterning cues provided by heparin-binding VEGF-A control blood vessel branching morphogenesis. Genes Dev. 16:2684-2698.

45. Gerhardt, H., et al. 2003. VEGF guides angiogenic sprouting utilizing endothelial tip-cell filopodia. J. Cell Biol. 161:1163-1177.

46. Soriano, P. 1994. Abnormal kidney development and hematological disorders in PDGF beta-receptor mutant mice. Genes Dev. 8:1888-1896.

47. Tidhar, A., et al. 2001. A novel transgenic marker for migrating limb muscle precursors and for vascular smooth muscle cells. Dev. Dyn. 220:60-73. 